Paidéia, 2002, 12(23), 5-17

\title{
AS APTIDÕES NA DEFINIÇÃO E AVALIAÇÃO DA INTELIGÊNCIA: O CONCURSO DA ANÁLISE FATORIAL ${ }^{1}$
}

Leandro S. Almeida ${ }^{2}$

Universidade do Minho

\begin{abstract}
RESUMO: Um dos percursos seguidos pela Psicologia na definição e avaliação da inteligência identifica-se com as tentativas de fixação do número das aptidões e, no caso de defesa de um número plural de aptidões, com a questão da eventual independência ou interdependência das mesmas aptidões. A análise fatorial, primeiro exploratória e agora confirmatória, tem sido o método por excelência para fundamentar este percurso, mesmo sem nunca conseguir dar razão a uns e retirá-la a outros. Não sendo o único modelo teórico de abordagem da inteligência pela Psicologia, certo que detêm um papel importante na investigação nesta área e, sobretudo, cabe-lhe a construção e a validação dos instrumentos usados pelos psicólogos nos vários domínios da sua prática profissional. Mesmo que criticada por se centrar mais nos produtos do que nos processos cognitivos inerentes aos desempenhos, a abordagem fatorial mantêm-se atual e produtiva na psicologia, e muito concretamente na área da inteligência.
\end{abstract}

Palavras-chave: Inteligência, Aptidões, Psicometria, Tests

\section{APTITUDES IN THE DEFINITION AND EVALUATION OF THE INTELLIGENCE: CONTRIBUTIONS OF FATOR ANALYSIS}

\begin{abstract}
One part of the research in psychology about the definition and evaluation of the intelligence concerns the fixation of the number of the aptitudes and, in the case of a plural number of aptitudes, if these are independent or interdependent. The fator analysis, first exploratory and now confirmatory, has been the method par excellence, even without never to appoint which theory is correct. Not being the only theoretical approach of study intelligence by psychology, it had an important role in the research in this area and, above all, it is important to recognize its contribution in tests construction and validation for several domains of professional practice of psychologists. Even if criticized because its tendency to concentrate more in the products than in the inherent cognitive processes, the fatorial approach stays actual and productive in the psychology, and namely in the area of the intelligence.
\end{abstract}

Key-words: Intelligence, Aptitudes, Psychometry, Tests

O termo "inteligência" é utilizado com demasiada freqüência, provavelmente sem nos interrogarmos o tempo suficiente sobre o seu real significado. Quanto ao conceito, podemos concordar com Jensen (1969) ao afirmar que "intelligence, like electricity, is easier to measures than to define", ou nas palavras da Anastasi (1990, p. 5) "the term intelligence has acquired too many excess meaning that obfuscate its nature". Certo que estamos face a um conceito ou construto que não desfruta de consenso, mesmo entre os especialistas na área. A controvérsia é grande, referindo Hunt $(1999$, p. 3) "I believe that much

\footnotetext{
${ }^{1}$ Artigo recebido para publicação em abril de 2002; aceito em julho de 2002

${ }^{2}$ Endereço para correspondência: Leandro S. Almeida, Faculdade de Psicologia e Ciências de Educação. Universidade do Minho Braga, Portugal. E-mail: leandro@iep.uminho.pt
}

of this noise is just that, noise". Mesmo assim, assume-se com algum acordo que a inteligência tem sobretudo a ver com a capacidade de pensar e de resolver problemas por parte dos indivíduos (Nickerson, Perkins \& Smith, 1994). Se pensarmos nas diferentes características humanas, a inteligência reporta-se às suas capacidades e habilidades. Howe $(1997$, p. 1) afirma que "Being intelligent matters; it makes a big difference to human lives" ou, dizendo de outra forma, observa-se que os indivíduos se diferenciam quanto à forma e quanto à capacidade para realizar tarefas intelectualmente exigentes.

Estas diferenças humanas de desempenho instituiram-se em objeto e justificação de algumas teorias sobre a inteligência e, mais ainda, sobre as 


\section{Leandro S. Almeida}

provas psicológicas propostas para a sua avaliação (Almeida \& Buela-Casal, 1997). Referimo-nos, sobretudo, à abordagem diferencial ou fatorial da inteligência (Almeida, 1994). Não se tratando, logicamente, da única corrente de definição e avaliação da inteligência (veja-se, por exemplo, a abordagem desenvolvimental e a abordagem da teoria do processamento da informação), parece-nos no entanto a mais popular e difundida entre os profissionais da área.

Neste artigo, tomando um enfoque histórico, apresentam-se as teorias mais relevantes na definição e descrição operacional da inteligência ao nível das aptidões. Neste quadro, como veremos, a discussão organiza-se em torno de duas questões associadas: (i) a existência de uma ou várias aptidões na definição da estrutura da inteligência, e (ii) uma organização horizontal ou vertical, em termos de importância das aptidões entre si, no caso dos modelos que propõem mais que uma aptidão. Todo este debate, como veremos, recorre à aplicação de extensas baterias de testes e à análise das dimensões subjacentes às respectivas intercorrelações através da análise fatorial (exploratória numa primeira fase e, mais recentemente, confirmatória). Estaremos particularmente atentos às implicações de tais teorias, sobretudo em relação à tipologia de provas psicológicas que propõem para a avaliação da inteligência.

\section{Inteligência como aptidão singular}

As primeiras abordagens da inteligência assumiram-na na sua unicidade. Se quisermos, buscava-se a inteligência na sua essência e não as várias aptidões que a poderiam diferenciar. Este movimento assente na unicidade da inteligência teve, no entanto, duas formas diferentes de expressão: (i) os que defendem a integração de funções cognitivas diversas num potencial ou quociente de inteligência (teoria da inteligência compósita), e (ii) os que propõem um elemento básico e comum a todas as atividades cognitivas (teoria do fator geral ou $g$ ).

\section{Teoria da inteligência compósita}

As primeiras concepções de inteligência faziam referência à capacidade associada à discriminação sensorial, ao tempo de reação e à coordenação sensório-motora (Almeida \& Buela-Casal, 1997). Estas concepções datam do final do século XIX, constituindo Sir Francis Galton um nome de referência ao conceber a capacidade intelectual como uma manifestação das capacidades mais básicas de discriminações sensoriais. A capacidade intelectual do sujeito refletiria a sua destreza e o grau de complexidade associativa nessas funções básicas, originando, então, a utilização de provas sensoriais e motoras na avaliação da inteligência. Galton, neste enquadramento teórico, construiu uma escala métrica, cujos testes avaliavam traços físicos, acuidade sensorial, força muscular e tempos de reação, entre outras capacidades sensório-motoras simples. Esta concepção da inteligência, e da sua mensurabilidade, foi continuada nos Estados Unidos com os trabalhos de James McKeen Cattell (Almeida, 1988; Nickerson, Perkins \& Smith, 1994; Richardson, 1991).

Algumas alterações relevantes vieram, entretanto, a ocorrer. Referimo-nos ao trabalho de Alfred Binet em França (Binet \& Simon, 1905). Para este autor, as diferenças intelectuais dos indivíduos decorrem de funções mentais mais complexas, por exemplo, memória, imaginação, atenção, compreensão ou apreciação estética, distanciando-se dos seus antecessores e sugerindo a avaliação de processos mentais superiores em alternativa às funções sensório-motores (Nickerson, Perkins \& Smith, 1994). O objetivo educativo de identificar as crianças mal sucedidas na escola por razões intelectuais leva-o à construção, em 1905, da Escala de Inteligência BinetSimon, assumida como um marco histórico na avaliação da inteligência (Almeida \& Buela-Casal, 1997).

Na sua versão inicial, a Escala de Inteligência Binet-Simon era constituída por 30 itens, distribuídos por ordem crescente de dificuldade e dirigida a crianças dos 3 aos 12 anos (Almeida, 1988; Richardson, 1991). Os itens estavam agrupados de acordo com a proporção de acertos das crianças por faixas etárias. Com este procedimento surge a noção de "Idade Mental" (desenvolvimento mental) que, associada à noção de "Idade Cronológica", permite o cálculo do QI (QI de razão), ou a percepção de um desenvolvimento intelectual normal, superior ou inferior por referência à idade do sujeito.

Refira-se que a escala de Binet-Simon foi re- 
vista em 1908 e em 1911, sendo adaptada por Louis Terman nos Estados Unidos, passando a ser conhecida por Escala Stanford-Binet e objeto de sucessivas revisões. Em França, os trabalhos de Zazzo e colaboradores conduziram às revisões de $1949 \mathrm{e}$ 1966, passando a designar-se Nova Escala Métrica da Inteligência (Zazzo, Gilly \& Verba-Rad, 1966).

O trabalho de Binet teve bastante impacto na psicologia. Uma definição assente nas funções cognitivas superiores conduziu à inclusão de tais funções nos itens das escalas de avaliação da inteligência. Por sua vez, a perspectiva integral do desenvolvimento e do funcionamento cognitivo do indivíduo justifica a opção por medidas globais do potencial cognitivo, como por exemplo o Q.I. (Anastasi, 1990). Assim, estas escalas supõem a avaliação de uma inteligência compósita, integrando de forma harmônica um conjunto heterogêneo de funções cognitivas.

A importância desta proposta de definição e de avaliação da inteligência prolongou-se no tempo, tornando-se bastante popular na psicologia. Isto explica, aliás, a construção de outras escalas similares, nomeadamente as escalas de Wechsler, ou seja, a WISC Wechsler Intelligence Scale for Children (1949), a WAIS - Wechsler Adult Intelligence Scale (1955) e a WPPSI - Wechsler Preschool and Primary Scale of Intelligence (1967). Incluem-se neste grupo, ainda, as escalas de Kaufman, como seja, a K-ABC - Kaufman Assessment Battery for Children (Kaufman \& Kaufman, 1983) e a KAIT - Kaufman Adolescent and Adult Intelligence Test (Kaufman \& Kaufman, 1993), por exemplo.

\section{Teoria do fator geral}

A par da popularidade das concepções e provas anteriores, importa referir que os avanços na estatística e, sobretudo, nos procedimentos de análise fatorial justificaram o aparecimento de outros modelos teóricos apoiados na análise de dados empíricos. Alguma legitimidade emergia para se buscar o fundamento das correlações entre várias provas e desempenhos cognitivos - os fatores ou estrutura interna da mente humana. As diferenças interindividuais nas habilidades cognitivas teriam a sua explicação nesses fatores ou dimensões internas, assumidos então como estruturantes da inteligência humana. Nascia, assim, uma abordagem da inteligência designada por fatorial (dimensões internas), psicométrica (testes, medida) ou diferencial (diferenças individuais), designações paralelas para descrever esta nova corrente (Almeida, 1988). Estes desenvolvimentos teóricos, como veremos, conduziram à construção de diferentes provas cognitivas procurando desse modo representar as aptidões específicas que se queriam avaliar. Mesmo que nem todos os estudos fatoriais defendam uma concepção multifacetada da inteligência, certo que grande parte do investimento havido na abordagem fatorial visava definir outras dimensões cognitivas complementares à perspectiva mais global, de algum modo traduzida no conceito de QI.

A maior ênfase na definição e avaliação de aptidões diferenciadas para a descrição da inteligência pode ter diversas justificações. Por um lado, o reconhecimento crescente da variabilidade intra-individual na realização dos testes de inteligência, 0 que acaba por não ser devidamente ponderado em provas cuja lógica é a obtenção de uma nota unitária. Esta nota, compósita por natureza, mostrava-se internamente pouco consistente, justificando a eliminação de determinados itens que, mesmo podendo traduzir comportamentos intelectuais pertinentes, não se apresentavam satisfatoriamente correlacionados com o conjunto escala. Em segundo lugar, e talvez mais determinante ainda, verificou-se um recrutamento progressivo dos psicólogos para atividades de orientação vocacional e de seleção de candidatos nos campos industrial e militar. Esta demanda social acelerou a criação de testes para a avaliação das múltiplas aptidões. Por último, importa mencionar a popularidade da análise fatorial na investigação psicológica. Ela providenciou as análises empíricas de apoio aos modelos teóricos de definição da inteligência e à construção das baterias multiaptidões. A análise fatorial instituiu-se, com efeito, na ferramenta por excelência dos investigadores na tarefa de identificar, agrupar e definir as diferentes aptidões (Anastasi, 1990). Infelizmente, tratando-se sobretudo de uma ferramenta exploratória de análise, possibilitou demasiadas teorias alternativas para a explicação dos mesmos dados.

\section{A concepção unitária de Spearman}

Spearman (1927) é autor da primeira teoria de inteligência baseada na análise estatística dos resultados nos testes. Em sua opinião, a inteligência poderia ser definida através de um fator simples (fa- 


\section{Leandro S. Almeida}

tor $g$ ) subjacente a todo o tipo de atividade intelectual e responsável pela maior parte da variância encontrada nos testes. Ao mesmo tempo, em cada teste existiria um fator específico (fator $s$ ), não generalizável a todos os testes (Almeida, 1988; Almeida \& Buela-Casal, 1997; Ribeiro, 1998).

Os dois fatores teriam origem distinta. $O$ fator geral dependeria de uma energia mental essencialmente biológica e inata, enquanto os fatores específicos dependeriam da aprendizagem, ou seja, seriam treináveis e educáveis, e ativados pelo fator $g$ (Almeida, 1988; Ribeiro, 1998). Como todas as atividades intelectuais partilhavam um único fator comum $(g)$ e os fatores específicos seriam singulares de cada atividade, uma correlação positiva entre dois desempenhos era atribuída ao fator geral. Quanto mais duas atividades estivessem saturadas em $g$, maior seria a correlação entre ẹlas, ocorrendo a situação inversa entre testes muito específicos.

A definição operativa do fator $g$ foi feita através de três leis de construção de conhecimento: (i) a apreensão da experiência; (ii) a edução de relações; e (iii) a edução de correlatos (Almeida, 1988). A sua avaliação seria mais bem conseguida através de testes que evitassem itens reportados a conhecimentos dos indivíduos, ou itens reportados a funções cognitivas muito específicas, por exemplo a percepção e a memória. A edução de relações e de correlatos seria melhor avaliada por meio de itens envolvendo o raciocínio indutivo e dedutivo. Assim sendo, ainda hoje os testes de fator $g$ enfatizam um conteúdo figurativo-abstrato dos itens, a novidade da tarefa (itens) e a centração nos processos de raciocínio. Referimo-nos, a título de exemplo, ao teste das Matrizes Progressivas de Raven, ao teste D48 de Pichot ou aos testes de Cattell (Almeida, 1994).

\section{A inteligência diferenciada em aptidões}

Ao contrário da corrente dominante na Euro$\mathrm{pa}$, alguns psicólogos americanos defendiam a inteligência como uma constelação de diferentes habilidades, relativamente independentes entre si. Também aqui, temos autores que, defendendo várias aptidões não as interligam, por exemplo, em função de influências recíprocas; e, temos autores que, defendendo várias aptidões cognitivas, não defendem a sua total autonomia, antes as seqüencializam numa cadeia hierárquica de interações e reciprocidades.

\section{As aptidões autónomas entre si}

Thurstone (1931) postula a existência de um determinado número de aptidōes primárias independentes entre si, e que explicariam o desempenho intelectual dos sujeitos. Mais tarde, defende que o fator geral é um artefato estatístico que descreve a estrutura da inteligência de uma forma muito pobre e nem sempre observável (Thurstone, 1938). Avança, então, com a idéia de que a inteligência é melhor compreendida como um conjunto de habilidades mentais primárias, isto é, por um conjunto de sete fatores independentes entre si: $\mathrm{V}$ - compreensão verbal, $\mathrm{W}$ - fluência verbal, $\mathrm{N}$ - aptidão numérica, $\mathrm{S}$ - aptidão espacial, $\mathrm{R}$ - raciocínio, $\mathrm{P}$ - velocidade perceptiva, e M- memória (Almeida, 1988; Ribeiro, 1998; Richardson, 1991; Sternberg \& Prieto, 1997). Por curiosidade, importa referir que o autor inicia a sua formulação por um modelo de nove fatores (Brody \& Brody, 1976; Horn \& Noll, 1994), passando, posteriormente, a sete dado que dois deles não eram suficientemente diferenciados ( $R$ - raciocínio aritmético e $\mathrm{D}$ - dedução viriam a ser abarcados no fator R- raciocínio). No quadro I descrevemos os fatores identificados por Thurstone.

Quadro I: Descrição dos fatores identificados por Thurstone

\begin{tabular}{|c|c|}
\hline Fator & Caracterização do fator \\
\hline Espacial $(S)$ & $\begin{array}{l}\text { Capacidade de visualização de objetos num espaço bi- ou tri-dimensional. De uma maneira geral são } \\
\text { itens figurativos. }\end{array}$ \\
\hline $\begin{array}{l}\text { Velocidade } \\
\text { perceptiva }(\mathrm{P})\end{array}$ & $\begin{array}{l}\text { Capacidade de, rapidamente e com acuidade, visualizar pequenas diferenças ou semelhanças entre } \\
\text { um grupo de figuras. }\end{array}$ \\
\hline Numérico $(\mathrm{N})$ & Capacidade de lidar com números e efetuar rapidamente operações aritméticas simples. \\
\hline Compreensão verbal (V) & Capacidade de compreensão de idéias expressas através de palavras. \\
\hline Fluência verbal (W) & Capacidade de produzir rapidamente palavras a partir de instruções apresentadas. \\
\hline Memória (M) & $\begin{array}{l}\text { Capacidade de evocar estímulos, como por exemplo pares de palavras ou frases, anteriormente } \\
\text { apresentados. }\end{array}$ \\
\hline Raciocínio (R) & Capacidade de resolver problemas, apreendendo e aplicando princípios, leis ou transformaçōes. \\
\hline
\end{tabular}


Mesmo não sendo possível verificar empiricamente a total independência entre os fatores isolados, Thurstone defende que os mesmos reúnem especificidade suficiente para serem concebidos como unidades funcionais independentes, justificando as próprias diferenças intraindividuais num conjunto de testes (Thurstone \& Thurstone, 1941). No quadro desta teoria, emergiram várias baterias para a avaliação das aptidões intelectuais. Em primeiro lugar, importa mencionar a Primary Mental Abilities (PMA) do próprio Thurstone.. Duas outras baterias merecem ser referidas, mais concretamente a Differential Aptitudes Tests (DAT) e General Aptitude Test Battery (GATB).

Guilford $(1959,1967)$ foi um outro autor a defender a inteligência formada por várias aptidões autônomas entre si. $\mathrm{O}$ seu modelo representa uma alteração bastante significativa face aos modelos fatoriais anteriores, partindo de um quadro téórico prévio para o trabalho empírico e não da exploração fatorial de dados para a teoria. Por este fato, a sua teoria é bastante divergente das apresentadas pelos demais autores (Acereda \& Sastre, 1998; Brody \& Brody, 1976; Eysenck, 1979), recorrendo a três componentes na definição das diferentes aptidões: operação mental (processo cognitivo envolvido numa dada tarefa), conteúdo (tipo de informação em que a tarefa se expressa) e produto (forma final da informação ou resultado após a atividade mental do su- jeito). $\mathrm{O}$ autor, cruzando cinco tipos de operações; quatro tipos de conteúdos e seis tipos de produtos, formula 120 aptidões no seu modelo estrutural da inteligência descrito na figura 1 . Assim, constatamos a existência de (i) quatro operações cognitivas: avaliação (processo de análise das respostas possíveis de acordo com critérios lógicos), produção convergente (resolução de problemas envolvendo processos de indução e dedução de relações), produção divergente (resolução de problemas envolvendo a produção de várias soluções possíveis), memória (retenção e evocação da informação) e cognição (reconhecimento e compreensão da informação); (ii) quatro conteúdos: figurativo (informação na forma de imagens), simbólico (informação na forma de signos cuja significação decorre de códigos), semântica (informação decorrente do significado de palavras ou outros elementos) e comportamental (informação associada a pensamentos e sentimentos acerca do próprio indivíduo è dos outros); e (iii) seis produtos: unidades (partes de informação relativamente limitadas), classes (agrupamentos de informação em função de características comuns), relações (conexões entre itens de informação), sistemas (agrupamentos de unidades estruturadas segundo padrões interrelacionados), transformações (modificações ou definição de fases da informação) e implicações (conexão circunstancial entre itens devido à sua proximidade).
Figura 1:

Modelo da
estrutura da
inteligência de
Guilford (1967)

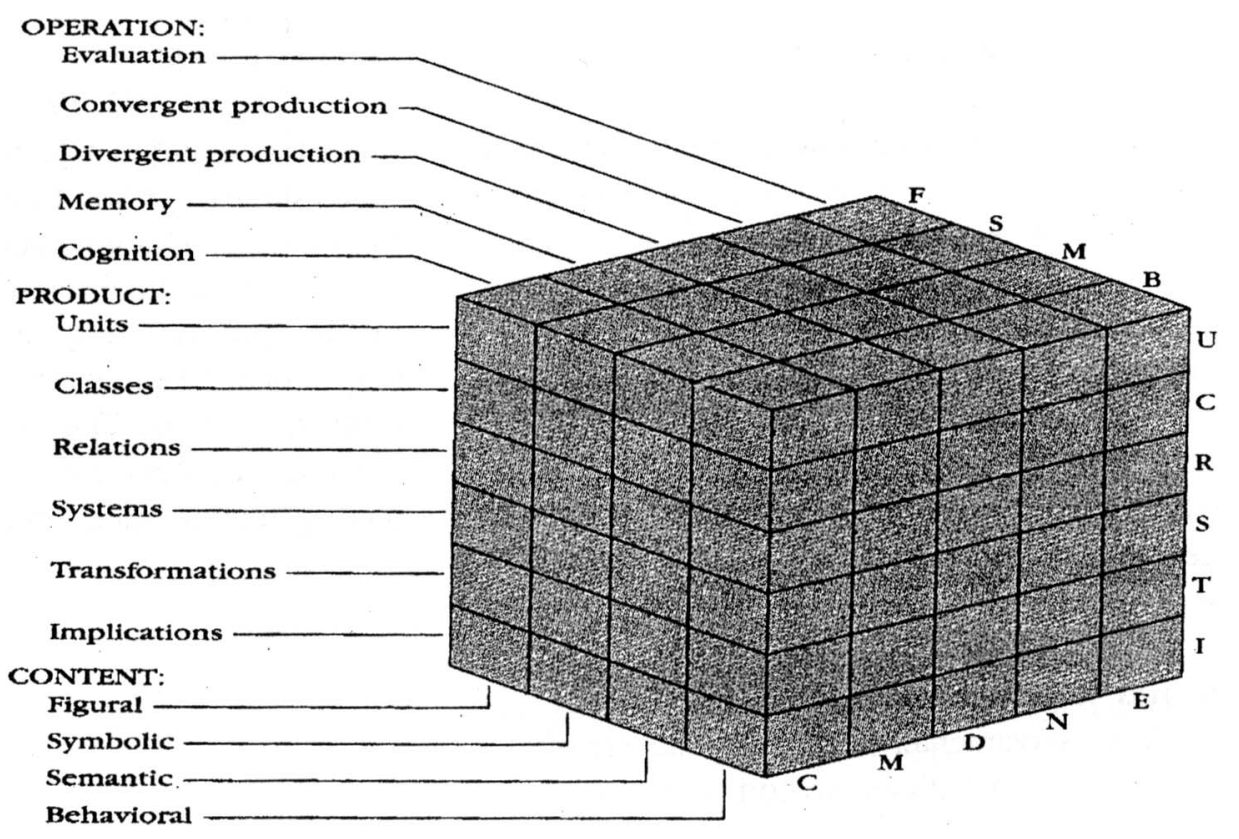




\section{Leandro S. Almeida}

Logicamente que um modelo tão complexo teria dificuldades na sua verificação empírica. Várias das funções cognitivas permanecem pouco definidas e, desde logo, sem provas específicas para a sua avaliação. Esta dificuldade complicar-se-ia no momento em que o próprio autor foi subdividindo algumas das categorias, chegando o modelo às 180 aptidões (Almeida, 1994). Por sua vez, algumas provas construídas pelo autor e seus colaboradores apresentam fracos índices de precisão e validade (Almeida, 1988; Armour-Thomas \& Gopaul-McNicol, 1998; Eysenck, 1979; Ribeiro, 1998).

A teoria de Guilford trouxe, também, alguns contributos interessantes ao estudo da inteligência (Almeida, 1988; Castelló, 1992). O primeiro tem a ver com a inclusão de processos cognitivos mais associados com a criatividade (produção divergente), complementares aos processos de raciocínio (produção convergente). $O$ segundo tem a ver com a inclusão do conteúdo comportamental entre os conteúdos que poderão diversificar as aptidões intelectuais dos indivíduos. Este conteúdo remete-nos para uma "inteligência social" que, por vezes, reaparece como questão importante no estudo da inteligência (Kihsltrom \& Cantor, 2000). Por último, o modelo SOI possui um valor heurístico interessante na identificação e diferenciação das aptidões (Guilford, 1988), o que tem as suas aplicações práticas. $O$ modelo sugere, por exemplo, uma grelha de análise dos curricuia e métodos de ensino nas escolas. A interligação proposta entre as capacidades cognitivas e as aprendizagens escolares facilitou a construção do "Structure-of-Intellect Learning Abilities Tests" (SOI-LA) (Meeker, Meeker \& Roid, 1985), sugerindo uma listagem bastante exaustiva de identificação dos diferentes processos cognitivos subjacentes ao trabalho intelectual. Isto, aliás, faznos citar a sua definição de inteligência como "uma coletânea sistemática de aptidões para 0 processamento, por vários modos, de diferentes tipos de informação" (Guilford, 1980).

\section{As teorias hierárquicas da inteligência}

Progressivamente, os autores mais significativos na área identificam-se com uma posição não unitária da inteligência, ou seja, defendem funções ou processos cognitivos diferenciados entre si. No entanto, acrescentam que tal diversidade de funções assenta em mecanismos cognitivos que promovem a respectiva interdependência e não a sua independência. No fundo, procura-se conciliar os pontos em confronto nas teorias de Spearman e de Thurstone (Almeida, 1998; Sternberg \& Powell, 1982). A idéia é a existência de fatores cognitivos funcionando em diferentes níveis de generalidade, isto é, uns mais gerais ou comuns a várias tarefas e outros mais específicos de uma dada tarefa. Decorre daqui a proposta de modelos hierárquicos sobre a estrutura da inteligência (Eysenck, 1979; Gustafsson, 1984, 1994).

Vernon (1950) propõe uma teoria em que, no topo da hierarquia, emerge o fator $\mathrm{g}$ de Spearman; no nível seguinte surgem dois fatores de grande grupo (verbal-educativo ou v:ed, e perceptivo-mecânico ou k:m); de seguida estes fatores subdividem-se em fatores de pequeno grupo ou secundários (bastante próximos dos fatores de Thurstone); e, finalmente, um conjunto bastante instável de fatores ainda mais específicos (Almeida, 1988; Anastasi, 1990; Ribeiro, 1998; Sternberg \& Prieto, 1997). Numa formulação posterior do seu modelo, Vernon (1950) propõe correlações entre fatores, especialmente os relacionados com a educação. As habilidades científicas e técnicas, por exemplo, estariam relacionadas com habilidades espaciais, mecânicas e numéricas (Anastasi, 1990):

Cattell $(1941,1943,1987)$ propõe uma segunda teoria hierárquica da inteligência, conhecida pela teoria da inteligência fluida (gf) e cristalizada (gc). Para Cattell, o fator $\mathrm{g}$ pode subdividir-se numa inteligência fluida (mais confinada ao próprio fator geral de Spearman) e numa inteligência cristalizada (capacidades assentes no uso das habilidades). Bastante na linha de Thurstone e de Guilford, Cattell defende a existência de dezenove fatores primários ou de primeira ordem (quadro II). 
Quadro II: Fatores primários identificados por Cattell

\begin{tabular}{|c|c|}
\hline Fator & Caracterizacäo \\
\hline Compreensão verbal (V) & Compreensāo de palavras e idéias. \\
\hline Aptidão numérica $(\mathrm{N})$ & Facilidade na manipulação de números. \\
\hline Fator espacial (S) & Compreensão de transformações de figuras num espaço bi ou tridimensional. \\
\hline Velocidade perceptiva (P) & Avaliaçāo rápida e eficiente de semelhanças ou diferenças em figuras. \\
\hline Velocidade de encerramento (Cs) & $\begin{array}{l}\text { Capacidade para reconstruir una imagem ou gestalt quando partes do estímulo estão } \\
\text { omissas. }\end{array}$ \\
\hline Raciocínio indutivo (I) & Raciocínio do específico para o geral. \\
\hline Memória associativa (Ma) & Aptidão para evocar unidades de informação fornecidas em pares \\
\hline Aptidão mecânica (Mk) & $\begin{array}{l}\text { Resoluçāo de situaçōes que envolvam os princípios da física, da mecânica ou outros } \\
\text { conhecimentos práticos. }\end{array}$ \\
\hline Flexibilidade de encerramento $(\mathrm{C})$ & $\begin{array}{l}\text { Aptidão para reconhecer determinado padrão de estímulos num determinado campo } \\
\text { perceptivo mais global. }\end{array}$ \\
\hline Amplitude de memória (Ms) & Evocação de informaçāo solta, recentemente apresentada. \\
\hline Ortografia (Sp) & Aptidão para reconhecer palavras com erros ortográficos. \\
\hline Avaliação estética (E) & Aptidão para detectar qualidades artísticas. \\
\hline Memória significativa (Mm) & Memorização de pares de elementos entrc os quais existe uma ligação com significado. \\
\hline Originalidade I (O1) & Fator que avalia a flexibilidade espontânea dos indivíduos. \\
\hline Fluência ideacional (Fi) & Capacidade de produzir idéias sobre um determinado tópico. \\
\hline Fluência de palavras (W) & Fator que respeita a produção rápida de palavras. \\
\hline Originalidade II (O2) & Aptidāo para combinar dois objetos afins para produzir um novo objeto. \\
\hline Precisão (A) & $\begin{array}{l}\text { Capacidade de movimentos rápidos e precisos envolvendo a coordenação sensório- } \\
\text { motora. }\end{array}$ \\
\hline Representação gráfica (Rd) & Capacidade de representação de estímulos através do desenho. \\
\hline
\end{tabular}

A partir de correlações entre estes fatores primários, Horn \& Cattell $(1966,1967)$ referem vários fatores de $2^{a}$ ordem (quadro III).

Quadro III: Fatores de 2a ordem (Horn \& Cattell, 1966)

\begin{tabular}{|l|l|}
\hline \multicolumn{1}{|c|}{ Fator } & \multicolumn{1}{|c|}{ Caracterização } \\
\hline Aptidão fluida (gf) & $\begin{array}{l}\text { Fator que representa a capacidade biológica do sujeitc ou a sua potência intelectual, e que se } \\
\text { traduz na apreensāo de relaçōes cumplexas (inferência, indução). }\end{array}$ \\
\hline Aptidão cristalizada (gc) & $\begin{array}{l}\text { Fator que representa a capacidade intelectual do sujeito associada ao seu processo de } \\
\text { aculturação. }\end{array}$ \\
\hline Capacidade de visualização (pv) & $\begin{array}{l}\text { Fator que representa a aptidão visual ou a resolução de problemas, envolvendo a imaginaçāo } \\
\text { de formas, sua rotação ou transformaçāo. }\end{array}$ \\
\hline Velocidade de realização (gs) & $\begin{array}{l}\text { Fator que representa a realização de tarefas intelectuais pouco complexas, apelando à } \\
\text { velocidade de realização. }\end{array}$ \\
\hline $\begin{array}{l}\text { Capacidade de evocação e de } \\
\text { fluência (gr) }\end{array}$ & Fator associado com a capacidade de evocação fácil e rápida de idéias, conceitos e palavras. \\
\hline
\end{tabular}

Brody e Brody (1976) referem que a divisão: entre inteligência fluida e cristalizada não se pode confundir com a suposição de dois fatores primários independentes. Ambas encontram-se intercorrelacionadas de forma moderada e positiva, o que não impede uma origem e natureza distintas: gf traduz uma aptidão essencialmente biológica, gc uma capacidade decorrente da aculturação dos indivíduos. Com efeito, o desenvolvimento e a aquisição das aptidões depende não só dos processos de aculturação (experiências educativas), mas também do grau de $g f$ de cada sujeito. A inteligência fluida funcionaria, assim, como o potencial intelectual do indivíduo.

Um terceiro modelo hierárquico de inteligência é proposto por vários autores recorrendo à análise fatorial confirmatória (Demetriou \& Efklides, 1994; Gustafsson, 1984; Gustafsson, Lindström \& Björck-Akesson, 1981; Undheim \& Gustafsson, 1987). Este modelo é conhecido pela sigla HILI, por associação com a metodologia de análise utilizada: $\mathrm{HI}$ - as duas primeiras letras da palavra Hierarchical, e LI de LISREL. Na figura 2 representamos este modelo com os seus componentes principais (Gustafsson, 1994).

No nível inferior, aparecem as aptidões 


\section{Leandro S. Almeida}

intelectuais defendidas por Thurstone. No nível intermédio, surgem fatores ditos gerais e já mais próximos da teoria de Horn-Cattell: Inteligência fluida (Gf) que abarca fatores primários tais como Indução (I), Raciocínio geral (R) e Cognição das relaçōes figurativas (CFR); Inteligência cristalizada (Gc) que abarca fatores primários como a Compreensão verbal (V) e a Cognição de relações semânticas (CMR); o fator geral de Visualização (Gv) que abarca a Visualização (Vz), Orientação espacial (S) e Flexibilidade de encerramento (Cf); o fator de geral velocidade (Gs) e precisão do processamento de informação em tarefas cognitivas de não alta complexidade cognitiva, e que abarca fatores primários como a aptidão Numérica $(\mathrm{N})$ e a aptidão Perceptiva (P); e o fator geral de Evocação (Gr) da informação estruturada em conhecimento, ou seja, na memória em longo prazo, e que abarca fatores primários como a Fluência de idéias ( $\mathrm{Fi}$ ) e a Fluência de palavras ( $\mathrm{Fw}$ ). No nível superior, encontramos um fator de inteligência geral $(g)$, no qual todas as aptidões de segunda ordem apresentam saturações elevadas (como a saturação de $g f$ é próxima da unidade, este fator $g$ pode assumir-se como equivalente à inteligência fluida).

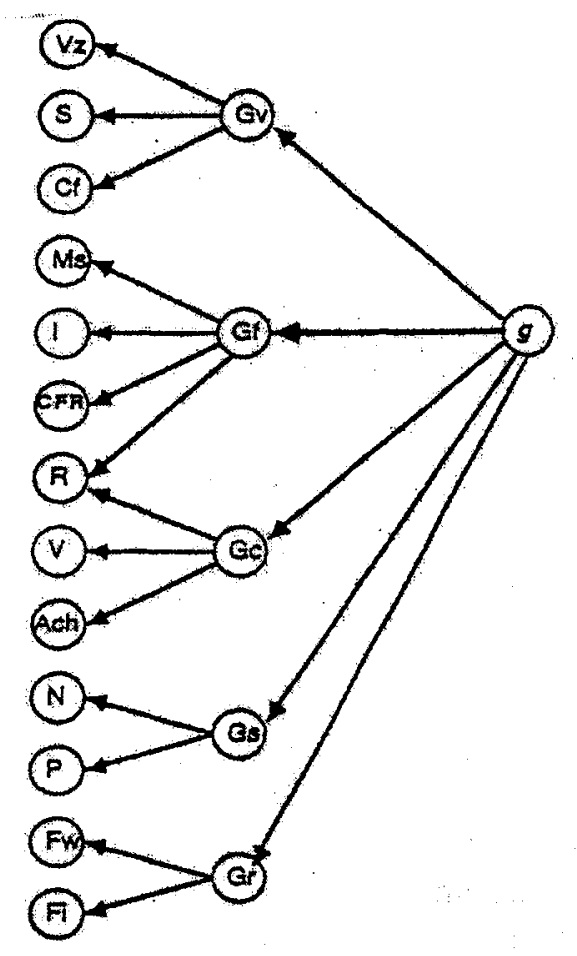

Figura 2:

Modelo hierárquico de Gustafsson (1994)
Um quarto modelo hierárquico é-nos proposto por Horn e Noll $(1994,1997)$, conforme indicado na figura 3. Este modelo postula nove aptidões: Inteligência fluida (Gf): Capacidade para apreender relações entre estímulos e para fazer inferências e compreender implicações entre os estímulos, particularmente em tarefas novas e complexas; Inteligência cristalizada $(\mathrm{Gc})$ : Capacidade para adquirir em extensão e profundidade o conhecimento da cultura dominante; Conhecimento quantitativo (Gq): Capa-. cidade para usar informação quantitativa e para manipular símbolos numéricos; Armazenamento e recuperação da informação à longo prazo (Glr): Capacidade para armazenar informação por um longo período de tempo e para a recuperar fluentemente mais tarde através de associações; Memória a curto prazo (Gsm): Capacidade para manter em consciência a informação e evocá-la uns segundos depois; Velocidade de processamento (Gs): Capacidade para rapidamente captar e responder a tarefas simples, em períodos de tempo curtos; Velocidade de decisão correta (CDS): Capacidade para rapidamente decidir e responder com acuidade a tarefas de dificuldade moderada; Processamento auditivo $(\mathrm{Ga})$ : capacidade para perceber padrões de sons, para manter a consciência da ordem e do ritmo de sons sob condições de distorção e distração, e para compreender relações entre diferentes grupos de sons; e, Processamento visual (Gv): Capacidade para perceber e manipular símbolos variando a forma e identificando variações na sua configuração espacial. Como se depreende, os fatores acompanham níveis seqüenciais de tratamento da informação, assumindo esta duas formas essenciais (figurativa e verbal). 


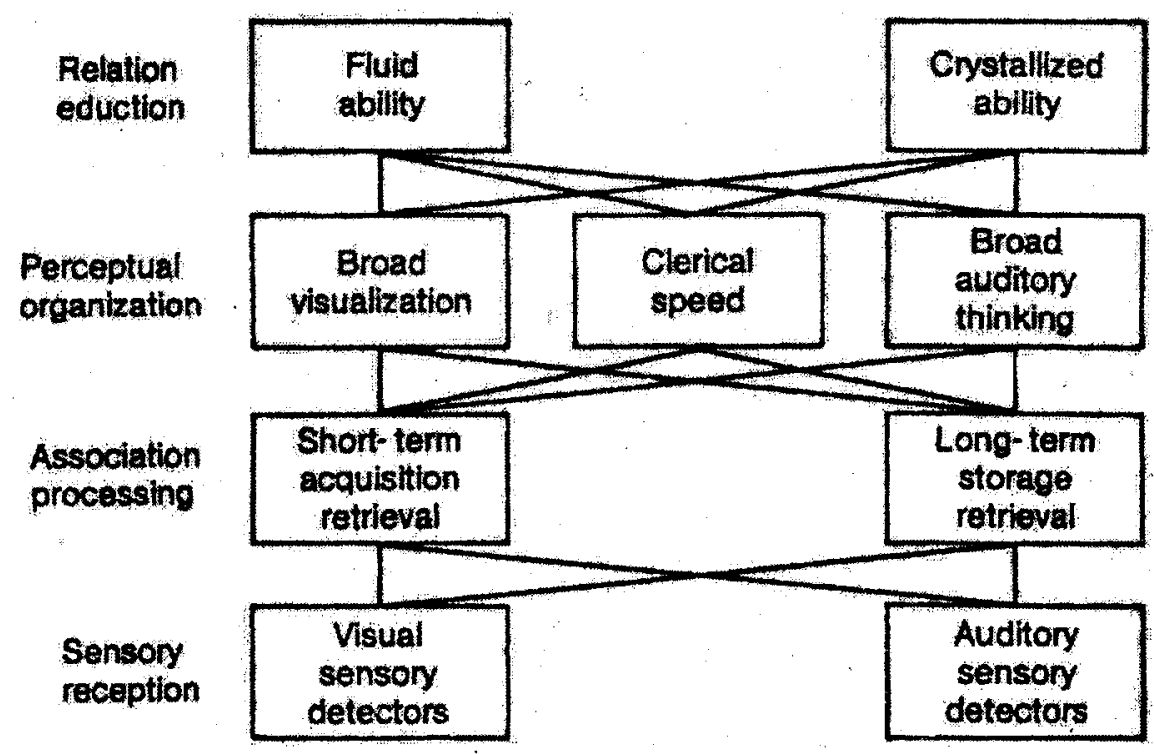

Figura 3: Modelo hierárquico da inteligência de Horn (1994)

Finalmente, ainda no quadro das teorias hierárquicas da inteligência, podemos referir a teoria dos três estratos de Carroll $(1993,1994)$, cujas linhas gerais estão descritas no quadro IV.
Como se depreende do termo "estrato", de novo estamos face a um modelo teórico assente em análises fatoriais de várias ordens.

Quadro IV: Teoria dos três estratos de Carroll (1994)

\begin{tabular}{|c|c|c|}
\hline $\begin{array}{c}\text { Estrato I } \\
\text { (Fatores de } \mathbf{1}^{\mathbf{2}} \text { Ordem) }\end{array}$ & $\begin{array}{c}\text { Estrato II } \\
\text { (Fatores de 2 } \text { 2 }^{\text {a }} \text { Ordem) }\end{array}$ & $\begin{array}{c}\text { Estrato III } \\
\text { (Fator de } 3^{\mathrm{a}} \text { Ordem) } \\
\end{array}$ \\
\hline $\begin{array}{l}\text { - Raciocínio Indutivo } \\
\text { - Raciocínio Quantitativo } \\
\text { - (..) }\end{array}$ & Inteligência Fluida & \multirow{8}{*}{ g (Inteligência Geral) } \\
\hline $\begin{array}{l}\text { - Compreensão Verbal } \\
\text { - Desenvolvimento da Linguagem } \\
\text { - (...) }\end{array}$ & Inteligência Cristalizada & \\
\hline $\begin{array}{l}\text { - Memória Associativa } \\
\text { - Memória Visual } \\
-(\ldots)\end{array}$ & $\begin{array}{c}\text { Memória e } \\
\text { Aprendizagem }\end{array}$ & \\
\hline $\begin{array}{l}\text { - Relações Espaciais } \\
\text { - Percepção de Formas } \\
\text { - (...) }\end{array}$ & Percepção Visual & \\
\hline $\begin{array}{l}\text { - Discriminação de Sons } \\
\text { - Discriminação Musical } \\
\text { - (...) }\end{array}$ & Percepçäo Auditiva & \\
\hline $\begin{array}{l}\text { - Originalidade } \\
\text { - Fluência Verbal } \\
-(\ldots)\end{array}$ & $\begin{array}{l}\text { Capacidade de } \\
\therefore \quad \text { Evocação }\end{array}$ & \\
\hline $\begin{array}{l}\text { - Aptidão Numérica } \\
\text { - Velocidade Perceptiva } \\
\text {-(...) }\end{array}$ & Velocidade Cognitiva & \\
\hline $\begin{array}{l}\text { - Tempos de Reação } \\
\text { - Velocidade de Processanento } \\
\text { Semântico } \\
\text {-(...) }\end{array}$ & Velocidade de Decisão & \\
\hline
\end{tabular}




\section{Leandro S. Almeida}

Nas análises que efetuo, Carroll (1994) diznos que encontrou, em geral, a existência destas capacidades e que, fazendo análises de segunda ordem (estrato II) das capacidades isoladas na primeira ordem (estrato I), encontrava os seguintes fatores gerais e respectivos fatores de primeira ordem associados: (i) Fator Gf (Inteligência fluida): Este fator abrange os processos e as capacidades básicas para o desempenho de atividades intelectuais que pouco dependem da aprendizagem e da aculturação. Este fator abarca no estrato I fatores como o raciocínio dedutivo e indutivo ou o raciocínio quantitativo, entre outros; (ii) Fator Gc (Inteligência cristalizada): Este fator representa o grau em que um sujeito é capaz de utilizar ou "investir" as capacidades básicas de forma a adquirir diversos conhecimentos, fruto de aprendizagens e da aculturação. Este fator abarca fatores como compreensão verbal, conhecimento lexical ou velocidade de leitura, entre outros; (iii) Fator Y (memória geral e aprendizagem): Capacidade geral de memorização de informação. Este fator inclui, a nível do estrato I, fatores como a memória de dígitos, memória associativa ou memória semântica, entre outros; (iv) Fator V (Percepção visual geral): Este fator representa uma capacidade geral para apreender e visualizar formas figurativas e espaciais. Este fator inclui, entre outros, fatores de visualização, relações espaciais ou velocidade perceptiva; (v) Fator U (Percepção auditiva geral): fator que influencia, na generalidade, os vários fatores de primeira ordem no domínio da percepção auditiva. Assim, abarca a discriminação do discurso, a aptidão musical ou a memória auditiva, por exemplo; (vi) Fator R (Capacidade geral de recuperação): Capacidade para recuperar rapidamente informação da memória a longo prazo, como demonstram os fatores envolvendo produção de idéias. Este fator abarca no estrato I fatores como a fluência de idéias e de palavras, a capacidade de nomeação ou a flexibilidade de respostas; e, (vii) Fator S (Velocidade): Este fator está presumivelmente relacionado com as capacidades que envolvem a velocidade da atividade cognitiva e do desempenho. Em termos do estrato I, este fator está associado quer à velocidade de processamento de informação quer aos tempos de reação a estímulos simples ou complexos. De referir que estes sete fatores gerais apresentam certa proxi- midade com algumas das inteligências propostas por Gardner (1983), muito embora a sua teoria não se apóie ou enquadre nos estudos fatoralistas.

\section{Considerações finais}

Um dos contributos sociais da investigação psicológica situa-se no domínio das teorias e dos testes na área da inteligência. Esta variável afeta os comportamentos humanos e, sobretudo, os seus níveis e formas de desempenho (Noronha, Sbardelini \& Sartori, 2001). O conhecimento científico das diferenças humanas neste domínio possibilitam decisões mais acertadas, por exemplo em contextos de orientação vocacional e de seleção profissional, mesmo que nem sempre unanimemente aceites. Ninguém gostaria de ser conduzido por um motorista com fracas habilidades espaciais... por muitos desempregados que existissem querendo ter nesta atividade profissional o seu sustento familiar.

A Psicologia, através de vozes distintas - às vezes bastante diversas para não dizermos controversas -, tem procurado definir as aptidões subjacentes ao desempenho humano e que, logicamente, explicam também as diferenças inter e intraindividuais a nível da sua inteligência. Com esta investigação, a Psicologia tem avançado na construção e validação de testes para a avaliação da inteligência, nas suas múltiplas formas, o que se traduz num produto genuinamente positivo dessa investigação. No entanto, temos que estar abertos a algumas críticas formuladas, e atentos ao fato de que, respondendo a necessidades sociais, este tipo de investigação não é neutra e pode proporcionar utilizações abusivas e socialmente injustas dos seus "produtos" científicos.

Um primeiro ponto a justificar alguma prudência passa pela própria inconsistência dos resultados da investigação. As ferramentas de análise usadas até ao presente viabilizam teorias diferentes e simultâneas, o que contradiz o objetivo da investigação científica no sentido de explicar e replicar os fenômenos em estudo. A análise fatorial sugere várias teorias quanto às formas como algumas capacidades intelectuais poderão existir e estar organizadas, não conseguindo apresentar uma solução única, muito menos em diferentes culturas e populaçōes. 
Para além disso, depende muito do tipo de testes que são utilizados para se fazer a matriz inicial de correlações, assim como do tipo de métodos de análise fatorial utilizados para extrair os fatores (Anderson, 1992).

Face ao exposto, aceitaremos que o recurso à análise fatorial exploratória e confirmatória não tem conseguido responder ao número, natureza e organização das aptidões humanas. Não the retirando o valor heurístico, não podemos assumir este método como "decisório" neste debate. Assim, longe de um consenso, parece-nos existir um acordo progressivo dos autores em torno de uma concepção que salvaguarda a natureza plurifacetada da inteligência em termos das funções ou das aptidões que integra. Defende-se, também, que tais funções ou aptidões não são independentes entre si, sem com isso descaracterizar a sua especificidade e, até, o interesse na sua avaliação independente na prática psicológica (Nickerson, Perkins \& Smith, 1994).

Apos esta breve resenha histórica da perspectiva psicométrica, referimos que os testes utilizados na psicologia permitem obter três tipos de medidas (Quociente de Inteligência, fator $g$ e aptidões), sendo que as principais implicações destes três tipos de testes passam pelas capacidades diferenciadas de apoiarem um diagnóstico de deficiências cognitivas e a análise de diferenças individuais tendo em vista as decisões ao nível do tratamento educativo diferenciado. Desta forma, avaliar a inteligência através de testes estandardizados assume que a execução de uma tarefa depende de uma série de habilidades implícitas na dita tarefa, denominando as habilidades como fatores (Sternberg \& Prieto, 1997).

A maioria dos testes psicométricos contemporâneos são baseados nas teorias multifatoriais, apesar de não seguirem, usualmente, as evoluções mais recentes havidas em tais teorias. Com efeito, os testes psicológicos têm evoluído pouco, possivelmente uma das "tecnologias" que sofreu menos inovação (Sternberg \& Kaufman, 1996). A generalidade destes instrumentos assentam a análise dos desempenhos nos resultados finais obtidos e dão pouca atenção aos processos e às estratégias seguidos pelos sujeitos na sua realização. Claro está que os problemas no uso dos testes não se centram exclusivamente neles mas nos seus utilizadores. Importa que o psicólogo seja melhor que os próprios testes, e não lhes fique refém nas suas decisões profissionais.

A controvérsia em torno dos testes de aptidão não é apenas científica (Gordon, 1988), por exemplo questiona-se frequientemente a pretensa e falsa neutralidade social dos testes que alguns autores pretendem defender. A questão mantêm toda a atualidade, mesmo num mundo em que o poder econômico e os meios de comunicação asseguram uma maior universalização dos produtos e formas de pensar. Como os demais produtos culturais, os testes adequam-se mais a determinados grupos, sendo da responsabilidade dos utilizadores saber distinguir o que é aptidão em cada contexto sócio-cultural e como pode, ou não, determinado teste adequar-se à sua avaliação (Roazzi, Spinillo \& Almeida, 1993). Como nas demais áreas da ciência e da tecnologia, um instrumento não pode suplantar nem substituir as competências técnicas do profissional que o utiliza. Esta ilação aplica-se quer ao nível da investigação quer, e sobretudo, da prática. Se isto tem cabimento nas ciências ditas exatas, por maioria das razões fará sentido na psicologia dada a idjossincrasia das suas situações e problemas. É, pois, uma área fundamental na formação graduada e pós-graduada dos psicólogos.

\section{Referências Bibliográficas}

Acereda, A. \& Sastre, S. (1998). La superdotación. Madrid: Editorial Sínteses.

Almeida, L.S. (1988). Teorias da inteligência. Porto: Edições Jornal de Psicologia.

Almeida, L.S. (1994). Inteligência: Definiçāo e medida. Aveiro: CIDInE.

Almeida, L.S. \& Buela-Casal, G. (1997). Evaluación de la inteligencia general. In G. Buela- Casal \& J.C. Sierra (Eds.), Manual de evaluación psicológica: Fundamentos, técnicas y aplicaciones. Madrid: Siglo XXI de España Editores.

Anastasi (1990). Psychological testing. New York: MacMillan.

Anderson, M. (1992). Intelligence and development: A cognitive theory. Oxford; Blackwell.

Armour-Thomas, E. \& Gopaul-McNicol, S. (1998). Assesssing intelligence: Applying a bio-cultural 


\section{Leandro S. Almeida}

model. California: SAGE Publications.

Binet, A. \& Simon, T. (1905). Méthodes nouvelles pour le diagnostic du niveau intellectuel des anormaux. Année Psychologique, 11, 191-244.

Brody, E.B. \& Brody, N. (1976). Intelligence: Nature, determinants and consequences. New York: Academic Press.

Carroll, J.B. (1993). Human cognitive abilities. Cambridge: Cambridge University Press.

Carroll, J.B. (1994). Cognitive abilities: Constructing a theory from data. In D. K. Detterman (Ed.), Current topics in human intelligence. Norwood: Ablex.

Castelló, A. (1992). Concepto de superdotación y modelos de inteligência. In Y. Benito (Ed.), Desarrollo y educación de los niños superdotados. Salamanca: Amaní.

Cattell, R.B. (1941). Some theoretical issues in adult intelligence testing. Psychological Bulletin, 38, 592.

Cattell, R.B. (1943). The measurement of adult intelligence. Psychological Bulletin, 40, 153193.

Cattell, R.B. (1987). Intelligence: Its structure, growth and action. Amsterdam: North Holland.

Demetriou, A. \& Efklides, A. (Eds.) (1994). Intelligence, mind, and reasoning: Structure and development. Amsterdam; North-Holland.

Eysenck, H.J. (1979). The structure and measurement of intelligence. New York: Springer Verlag.

Gardner, H. (1983). Frames of mind: The theory of multiple intelligences. New York: Basic Books.

Gordon, R.A. (1988). Thunder from the left. Review of "Storm over biology: Essays on science, sentiment, and public policy". Academic Questions, 1, 74-92.

Guilford, J.P. (1959). Three faces of intellect. American Psychologist, 14, 469-679.

Guilford, J.P. (1967). The nature of human intelligence. New York: McGraw-Hill.

Guilford, J.P. (1980). Fluid and crystallized intelligence: Two fancifull concepts. Psychological Bulletin, 88 (2), 406-412.

Guilford, J.P. (1988). Some changes in the structure- of-intellect model. Educational and Psychological Measurement, 48, 1-4.

Gustafsson, J.E. (1984). A unifying model for the structure of intellectual abilities. Intelligence, 8 , 179-204.

Gustafsson, J.E. (1994). Hierarchical models of intelligence and educational achievement. In A. Demetriou \& A. Efklides (Eds.), Intelligence, mind, and reasoning: Structure and development. Amesterdam: North-Holland.

Gustafsson, J.E.; Lindström, B. \& Björck-Akesson, E. (1981). A general model for the organization of cognitive abilities. Report from the Department of Education, University of Göteborg.

Horn, J.L. \& Cattel, R.B. (1966). Refinement and test of the theory of fluid and crystallized ability intelligence. Journal of Educational Psychology, 57(5), 253-270.

Horn, J.L. \& Cattel, R.B. (1967). Age differences in fluid and crystallized intelligence. Acta Psychologica, 26, 107-129.

Horn, J. \& Noll, J. (1994). A system for understanding cognitive capabilities: A theory and the evidence on which it is based. In D. K. Detterman (Ed.), Current topics in human intelligence. Norwood: Ablex.

Horn, J. \& Noll, J. (1997). Human cognitive capabilities: Gf- Gc theory. In D.P. Flanagan, J.L. Genshaft \& P.L. Harrison (Eds.), Contemporary intellectual assessment: Theories, tests, and issues. New York: The Guilford Press.

Howe, M.J.A. (1997).IQ in question: The truth about intelligence. London: SAGE publications.

Hunt, E. (1999). Intelligence and human resources: Past, present, and future. In, Ph. Ackerman, P.C. Kyllonen \& R.D. Roberts (Eds.), Learning and individual differences: Process, trait, and content determinants. Washington, DC: American Psychological Association.

Jensen, A.R. (1969). How much can we boost IQ and scholastic achievement? Harvard Educational Review, 39(1), 1-123.

Kaufman, A.S. \& Kaufman, N.L. (1983). Kaufman Assessment Battery for Children (K-ABC). 
Circle Pines, MN: American Guidance Service.

Kaufman, A.S. \& Kaufman, N.L. (1993). Kaufman Adolescent and Adult Intelligence Test (KAIT). Circle Pines: American Guidance Service.

Kihsltrom, J.F. \& Cantor, N. (2000). Social intelligence. In R. J. Sternberg (Ed.), Handbook of intelligence. Cambridge: Cambridge.

Meeker, M.N., Meeker, R. \& Roid, G.H. (1985). Structure-of-intellect learning abilities tests, Manual. Los Angeles: Western Psychological Services.

Nickerson, R.S., Perkins, D.N. \& Smith, E.E. (1994) (Eds.). Enseñar a pensar: Aspectos de la aptitud intelectual. Paidós: M.E.C.

Noronha, A.P.P., Sbardelini, E.T.B. \& Sartori, F.A. (2001). Análise da qualidade dos testes de inteligência publicados na Brasil. Psico-USF, 6(2), 95-104.

Ribeiro, I. (1998). Mudanças no desempenho e na estrutura das aptições: Contributos para o estudo da diferenciação cognitiva em jovens. Braga: Universidade do Minho.

Richardson, K. (1991). Understanding intelligence. Philadelphia: Open University Press.

Roazzi, A., Spinillo, A. \& Almeida, L.S. (1993). Definição e avaliação da inteligência: Limites e perspectivas. In L. S. Almeida (Ed.), Cognição e aprendizagem escolar. Porto: APPORT.

Spearman, C. (1927). The abilities of man. New York: MacMillan.

Sternberg, R.J. \& Kaufman, J.C. (1996). Innovation and intelligence testing: The curious case of the dog that didn't bark. European Journal of Psychological Assessment, 12 (3), 175-182.

Sternberg, R.J. \& Powell, J.S. (1982). Theories of intelligence. In R.J. Sternberg (Ed.), Handbook of human intelligence. Cambridge: Cambridge University Press.

Stemberg, R. \& Prieto, M.D. (1997). Evaluación de las habilidades de la inteligencia: Teoria triárquica de la inteligencia. In G. Buela- Casal \& J.C. Sierra (Eds.), Manual de evaluación psicológica: Fundamentos, técnicas y aplicaciones. Madrid: Siglo XXI de España Editores.
Thurstone, L.L. (1931). Multiple fator analysis. Psychological Review, 38, 406-427.

Thurstone, L.L. (1938). Primary mental abilities. Chicago: University of Chicago Press.

Thurstone, L.L. \& Thurstone, T.G. (1941). Fator studies of intelligence. Chicago: University of Chicago Press.

Undheim, J.O. \& Gustafsson, J.E. (1987). The hierarchical organization of cognitive abilities: Restoring general intelligence through the use of linear structural relations. Multivariate Behavioral Research, 22, 149-171.

Vernon, P.E. (1950). The structure of human abilities. London: Methuen.

Wechsler, D. (1949). Wechsler Intelligence Scale for Children. San Antonio: Psychological Corporation.

Wechsler, D. (1955). Wechsler Adult Intelligence Scale. San Antonio: Psychological Corporation.

Wechsler, D. (1967). Wechsler Preschool and Primary Scale of Intelligence. San Antonio: Psychological Corporation.

Zazzo, R., Gilly, M. \& Verba-Rad, M. (1966). Nouvelle Échelle Métrique de l'Intelligence. Paris: A. Colin. 
